\title{
Nanocomposite tubes for magneto-active devices
}

\author{
V. Guarino ${ }^{1}$, V. Iannotti ${ }^{2}$, G. Ausanio ${ }^{2}$, L. Ambrosio ${ }^{1}$, L. Lanotte Li, $^{1}$ \\ ${ }^{1}$ Institute of Polymers, Composites and Biomaterials (IPCB) National Research Council of Italy, Mostra d'Oltremare \\ Pad. 20, V.le J. F. Kennedy 54, I-80125 Naples, Italy \\ ${ }^{2}$ CNR-SPIN and Department of Physics 'E. Pancini', University of Naples 'Federico II', Piazzale V. Tecchio 80, I-80125 \\ Naples, Italy
}

Received 23 September 2019; accepted in revised form 2 December 2019

\begin{abstract}
Small polymeric ducts incorporating a ribbon-shaped mat of densely packed magnetic nanofibers have been manufactured via electrospinning by using a cylindrical manifold, alternately under rotation or static. The magnetic nanofibers mat is located on the side of the tube and aligned to the longitudinal axis using the assistance of a magnetic field. The designed methodology ensures that the magnetic particles are completely wrapped into a protective polymer shell. Experimental results demonstrate that the innovative confinement of magnetic nanofibers, forming a longitudinal ribbon on a tube side, confers a high and reversible transverse strain under a moderate magnetic field stimulus: a magnetic field gradient $\leq 30 \mathrm{mT} / \mathrm{mm}$, at a basic field intensity $<0.04 \mathrm{~T}$, induces a $40 \%$ decrement of the duct radius aligned with the magnetic force axis. In perspective, this is very attractive to fabricate magneto-active ducts suitable for microfluidic components, as well as biomedical devices to be applied in surgery and endoscopy.
\end{abstract}

Keywords: smart polymers, magnetic field-assisted electrospinning, magneto-active microtubes, elastomagnetic strain effects

\section{Introduction}

In the last decade, the production of electrospun fibers is experiencing significant improvements in the process technology (i.e., bubble or macromolecular electrospinning [1-3]) greatly contributing to the realization of advanced functional materials [4-8]. In particular, electrospun tube-shaped devices are assuming an increasing role in biotechnology [9] and in various biomedical applications such as vascular prosthesis [10], artificial ducts [11-15], bioactive ducts for nerves [16], tendon rupture repair [17], adhesions prevention [18], reinforced medical tubes [19], antimicrobial urinary catheters [20]. At the same time, the development of intelligent polymers that respond to small changes in environmental conditions with shape, physical, or chemical changes is achieving interesting improvements in micromechanics and biocompatible devices [21-24]. Moreover, a significant effort was employed for the development of smart polymer tubes capable of providing deformation with multiple degrees of freedom for applications in robotic manipulation [25, 26], displacement actuators [27], shape recovery components [28], $\mathrm{pH}$ control [29].

In this context, our investigation has been recently addressed to the fabrication and characterization of elastomagnetic nanostructured tubes consisting of magnetically functionalized polycarbonate-urethanes nanofibers, incorporating Nickel nanoparticles [30]. In this work, the process optimization in terms of incorporation and dispersion of magnetic particles into the fibers was reported, also clarifying the role of particle size and aggregation in the functionalities of magneto-active devices. These nanostructured tubes exhibited noteworthy smart properties, in particular, a large longitudinal strain under a moderate magnetic field. At the same time, taking

${ }^{*}$ Corresponding author, e-mail: lanotte@fisica.unina.it

(C) BME-PT 
advantage of previous pioneering studies on the combination of magnetic field with electrospinning process [31], a proper configuration of electric and magnetic fields was defined to agglomerate magnetic nanofibers, aligned along the line of maximum intensity of the magnetic force, thus forming nanofibrous wires with a high density of magnetic nanoparticles: this peculiar structure is the main responsible for high elastomagnetic deflection and elongation, very promising for electromechanical and biomedical devices [32].

Within the above-described state of the art, the production of programmed/aligned filiform fibers covers recent developments [33], and several methods of external assistance during electrospinning, also in cylindrical configuration [34], have been already used. Nevertheless, these previous studies have not yet been specifically addressed to the realization of a magnetic functionalization for the improvement of elastomagnetic sensitivity. This is just our general purpose: on the last aspect the main progress of the proposed procedure and the originality of the new features are based. In fact, in this investigation we design and produce nanocomposite tubes incorporating a thin longitudinal ribbon of magnetic equally oriented nanofibers, well-localized laterally on the duct. This innovative structure leads obtaining tubes that exhibit a remarkable transverse contraction (over $50 \%$ increase of the tube eccentricity with a $40 \%$ decrease in tube radius along the axis of external magnetic force application) under moderate magnetic stimulus (a magnetizing field gradient $\leq 30 \mathrm{mT} / \mathrm{mm}$ at a basic intensity $<0.04 \mathrm{~T}$ ). These new magnetically functionalized polymer tubes (MFPTs), could be very important to follow up the demand of tubular devices able to exhibit a transversal strain to solve very frequent obstruction problems in bio-medicine or for micro-electromechanical flow regulator devices, as it will be indicated with more detail in the conclusions.

In the following sections, the used materials, the experimental expedients and the steps of the employed methodology will be described. Then, results on the morphological, magnetic and, in particular, elastomagnetic strain characterization, will be reported to validate the achieved points:

a) Fabrication of an elastic polymer tube containing a longitudinal ribbon of magnetic nanofibers well incorporated inside by a programmed sequence of process conditions; b) Validation of the new composite tubes as sensitive actuators in prototypes capable of a significant transverse contraction under moderate magnetic stimuli.

\section{Materials, production and characterization method}

In agreement with previous works, composite nanofibers of Polycarbonate-urethanes (PCU), including Nickel nanoparticles (99,99\% Aldrich Chemical), as filling magnetic charge, were fabricated via electrospinning.

Among the large choice of potential polymer matrices (see for example references [35, 36]), PCU were selected for their high elastomagnetic sensitivity, bio-compatibility, and good elasticity. In fact, due to its peculiar chemical structure which includes three reactive components: a di-isocyanate, a soft segment - commonly an oligomeric macro-monomer -- and a chain extender, PCU show an unique combination of toughness, durability, flexibility, biocompatibility and bio-stability making it suitable for a wide range of applications in the biomedical field as for example functional support of damaged vessels [37], vascular prosthesis [38] and regeneration of connective tissues [39] exhibiting good elasticity performances and durability in vivo [40]. On the market, two different types of PCUs named CORETHANE (75D and $80 \mathrm{~A}$ ) are available. In our study we used latter (80A) because it shows a peculiar rubbery behavior conferred by the higher fraction of soft segments.

On the other side, Nickel nanoparticles (NPs) with a size $<200 \mathrm{~nm}$ have been used in order to ensure their incorporation in micrometric-sized fibers, preserving a remarkable magnetic moment. Other high-performance magnetic particles could be chosen [41-43], but the main reason we prefer Nickel is that it has already shown to have interesting elastomagnetic response in a polymeric matrix, as reported in previous works [30, 32, 44-47].

For the electrospinning process, a basic solution was prepared by dissolving Corethane $(15 \% \mathrm{w} / \mathrm{v})$ into a THF/DMF (7:3) until a complete homogeneity is achieved. In one-half of the PCU solution, the Ni nanoparticles were mixed manually for 20 minutes, in the right percentage to obtain a homogeneous viscous solution with a Ni content of $12 \%$ in volume. The pure PCU solution, as well as the NPs charged solution, were placed in a $5 \mathrm{ml}$ plastic syringe connected to an 18 Gauge needle. 
The electrospinning apparatus (Nanon01, MECC, Japan) has a simple configuration, as shown in Figure 1. The liquid jet is directed along the vertical axis and, taking into account the optimization already obtained for the production of nanofiber tubes and wires [30, 32], the following values of the set-up parameters were fixed: flow rate of $1 \mathrm{ml} / \mathrm{h}$, needle/collector gap equal to $12 \mathrm{~cm}$, voltage difference between collector and emitter equal to $18 \mathrm{kV}$. Moreover, the spinneret was translationally moved - at a rate of $1 \mathrm{~mm} / \mathrm{s}$ - along $35 \mathrm{~mm}$ width to improve fiber thickness homogeneity along the tube.

The production process was adapted to obtain polymeric tubes with two main requirements: (i) incorporation of magnetic nanofibers preferentially oriented along to the longitudinal axis of the tube and located in a lateral strip of the conduct; (ii) an optimal encapsulation of the magnetic nanoparticles into PCU casing. The first requirement is mandatory to obtain high transversal and flexural strains under external magnetic gradients stimuli, while the latter one to prevent any contamination of the magnetic NPs with outside environment, thus preserving the biocompatibility.

To achieve the prefixed goals, the process was performed in three successive operative steps:

1) fiber deposition from pure PCU solution onto the horizontal stainless steel mandrel $-3.0 \mathrm{~mm}$ in radius - with controlled rotation of $50 \mathrm{rpm}$.
(10 minutes). The rod was previously wrapped by an aluminum foil to facilitate the extraction procedure of the fibrous tube once collected.

2) fibre deposition of NPs/PCU solution (12 minutes) under process conditions as reported elsewhere [32]. In this case, the mandrel rotation was interrupted and two permanent $\mathrm{NdFeB}$ magnets $\left(30 \times 30 \times 6 \mathrm{~mm}^{3}\right)$, at a distance of 6 millimeters below the mandrel, were positioned as shown in Figure 1 to produce a horizontal magnetic field, transverse to the deposition axis, thus generating a layer of aligned magnetic nanofibers on the upper side of the collector rod, above the previous deposited layer of PCU fibers (Figure 1b, below).

3) fiber deposition from the pure PCU solution (10 minutes) to form a homogeneous external layer of pure PCU fibers onto the surrounding tube already formed in the previous two steps.

After the deposition, free MFPT samples were obtained by sliding the thin aluminum foil wrapped onto the metallic mandrel, put on the manifold as a holder for the deposited material, then removing the residual aluminum from the inner part of the conduits.

The side and cross-section images of the produced tubes were acquired by means of an optical magnification camera (TESLONG MS 100 photo-camera). The morphology of the nanofibers was investigated by SEM microscopy (FESEM, QUANTA200, FEI,

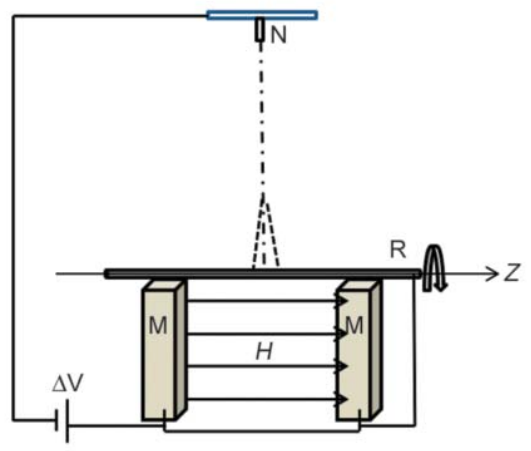

a)

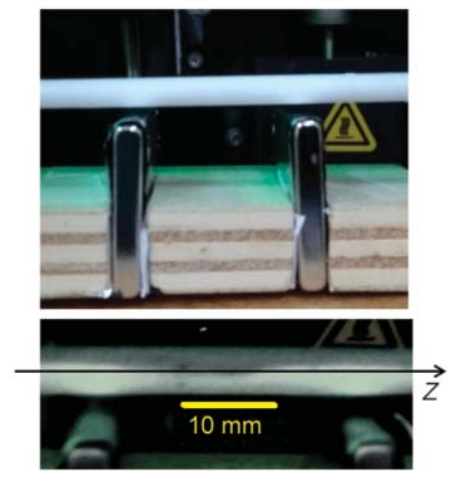

b)

Figure 1. a) Layout scheme of the electrospinning equipment. $\mathrm{N}=$ needle, $\mathrm{R}=$ cylindrical holder (that can be in the rotation or stopped), $\Delta \mathrm{V}=$ Voltage Generator, $\mathrm{M}=\mathrm{NdFeB}$ permanent magnets (maximum induction field on the polar surface: $0.4 \mathrm{~T}$ ) producing a static field $\mathrm{H}$ (which can be easily removed). The horizontal displacement of the needle was fixed at $35 \mathrm{~mm}$ to cover the distance between the middle points of the top surfaces of the magnets. b) Above: arrangement of the magnets under the PCU duct obtained after $1^{\text {st }}$ phase. Below: detail of the tube obtained after $2^{\text {nd }}$ phase with electrospun magnetic nanofibers (darker in color due to the NPs presence) forming a strip on the upper region. The magnets are fixed on a wooden base plate, coming out at a height of $1.5 \mathrm{~cm}$ (distance between the magnets $30 \mathrm{~mm}$ ). An aluminum sheet covers each magnet and goes down to contact the electrode at the base. The electric contact between the mandrel, the magnets and the aluminum foil is ensured by conductive wires. Thus, the electric field distribution is stabilized, ensuring that the magnets, the aluminum sheets on their surface and mandrel have the same potential. 
the Netherlands). Prior to the analysis, circular samples, mounted on metal tubes by using double-sided adhesive tape, were sputtered with a gold-palladium coating for about 20 seconds. SEM images were taken under high vacuum conditions $\left(10^{-7}\right.$ tor $)$ using the secondary electron detector (SED), at a working voltage of $5 \mathrm{kV}$.

The magnetic characterization of the permanent magnet was carried out by means of standard Hall Probes, while the magnetic response of the samples was characterized by measuring the differential magnetic permeability $\mu=\delta B / \delta H$ where $H$ is the magnetizing field applied parallel to the longitudinal axis of the tubular sample and $B$ is the respective longitudinal magnetic induction in the sample, obtained from the first magnetization curve. The first magnetization curve and its derivative were determined by using a Vibrating Sample Magnetometer (VSM, Oxford Instrument) - in dotation in our laboratory [48] - and its data processing facilities.

\section{Results and discussion}

\subsection{Morphological and magnetic analyses: preliminary studies}

A focus point to fabricate the MFPTs described above consists of the effectiveness of the magnetic field assistance to produce a ribbon of compact and aligned magnetic functionalized nanofibers (MFNs) by electrospinning a polycarbonate-urethane (PCU) + magnetic nanoparticles (MNPs) solution on a pure PCU tube - previously deposited.

To verify this magnetic field efficiency, a morphological characterization of magnetic nanofibers mats, as deposited on a pre-existent PCU tube, was performed in three different conditions: (a) cylindrical manifold held in rotation without the presence of longitudinal magnetization; (b) rotating cylindrical manifold and in the presence of a longitudinal magnetic field; (c) manifold in static condition and presence of longitudinal magnetization.

After the addition of another outer PCU layer, the final tubular samples are removed from the cylindrical aluminum support. The image of their cross-section provides a clear view of the macroscopic effects obtained keeping the support in rotation during the electrospinning of the MNFs (procedure (a) or (b), Figures $2 \mathrm{a}, 2 \mathrm{~b}$ ) - thus helically distributing the magnetic fibers over the entire surface of the polyurethane deposit, formed in the previous phase - or stopping the support itself and depositing the MNFs

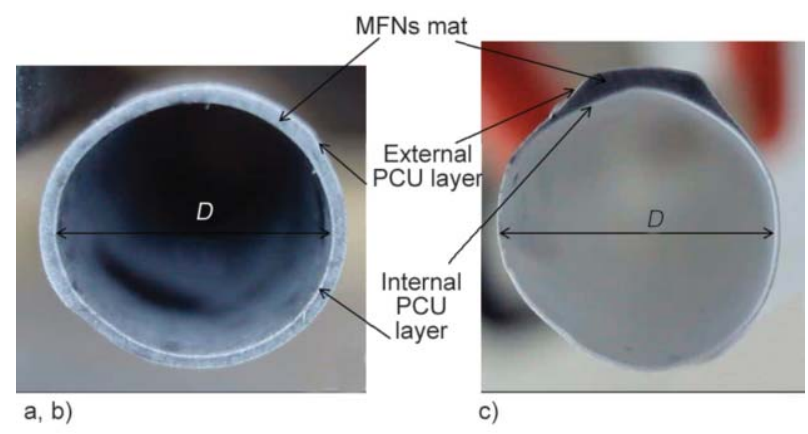

Figure 2. (a, b) Cross-section of the tubular sample obtained with procedure (b) (magnetic field-assisted electrospinning on rotating manifold). In the case of absence of magnetic field (procedure (a)) the crosssection image does not show difference, therefore only one figure representative of the two cases is reported. (c) Cross-section of a tubular sample produced according to the procedure (c) (magnetic field-assisted electrospinning on the fixed manifold). The cross-section diameter is $D \approx 6.3 \mathrm{~mm}$.

longitudinally and only on a side strip of the cylinder (procedure (c), Figure 2c).

In the case in which the distribution of the MFNs covers the entire circular crown (Figures 2a, 2b) the dilution of the nanofibers is high - this is also demonstrated by the fact that the nickel presence does not give gray color in the image of the section -. On the contrary, in the case of Figure 2c it is very evident the thickening of the MFNs that form a mat only on the section edge exposed to electrospinning of the NPs/PCU solution. Since the same cross-section configuration is detected along the entire length of the tube, the MFNs form a longitudinal ribbon densely loaded with nickel particles inside the wall of the small conduit.

In the case (a), also if the tube cross-section appears very similar to that of the case (b), a low pre-orientation of the fibers is expected. To better clarify the MNFs alignment inside the deposited mat, their morphology was investigated before the outer shell electrospinning. In Figure 3 we can see that the MFNs in condition (a) (Figure 3a) are inclined with respect the longitudinal axis $Z$ (Figure 1) as a consequence of the collector rotation, and this inclination is drastically reduced if longitudinal magnetic field is applied (Figure $3 b$ ): therefore, the magnetic action on the NPs, which try to align them towards the magnetic force lines, also produces an alignment of the fiber towards the $H$ axis, coincident with $Z$. This is validated by the MFNs image after electrospinning on the collecting manifold in static condition (Figure $3 \mathrm{c}$ ): the fibers are preferentially parallel to $H$. 


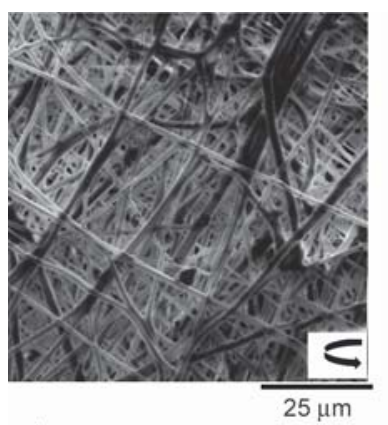

a)

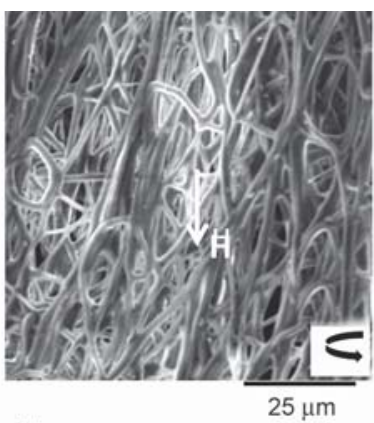

b)

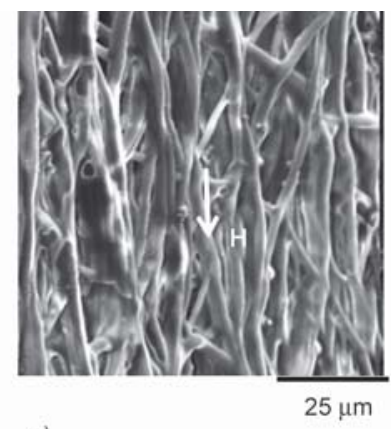

c)

Figure 3. SEM images of nanofibers deposited on rotating manifold without (a) and with (b) longitudinal magnetic field, and in static conditions with the presence of a longitudinal magnetizing field (c). The samples are all obtained after the PCU fibers electrospinning on the rotating collector for 5 minutes and subsequent electrospinning of PCU+Nickel nanoparticles for further 5 minutes in the three different methodologies (a), (b) and (c). The average fibers diameter, evaluated via image analysis was equal to (a) $1.1 \mu \mathrm{m}$, (b) $1.8 \mu \mathrm{m}$ and (c) $2.2 \mu \mathrm{m}$, respectively.

In the three investigated case the particle concentration in the used solution was fixed at $12 \%$ in volume, this because we have designed to evidence only the effect of particle orientation on the properties of the deposited fibers. An indirect experimental confirmation of the Ni nanoparticles orientation inside the fibers, due to magnetic field application during static or dynamic electrospinning, is obtained by comparing the behavior of the longitudinal differential magnetic permeability $\mu=\delta B / \delta H$ as defined in section 2 . The results obtained from magnetically functionalized cylindrical samples (20 $\mathrm{mm}$ in length) produced under conditions (a), (b) and (c) are summarized in Figure 4.

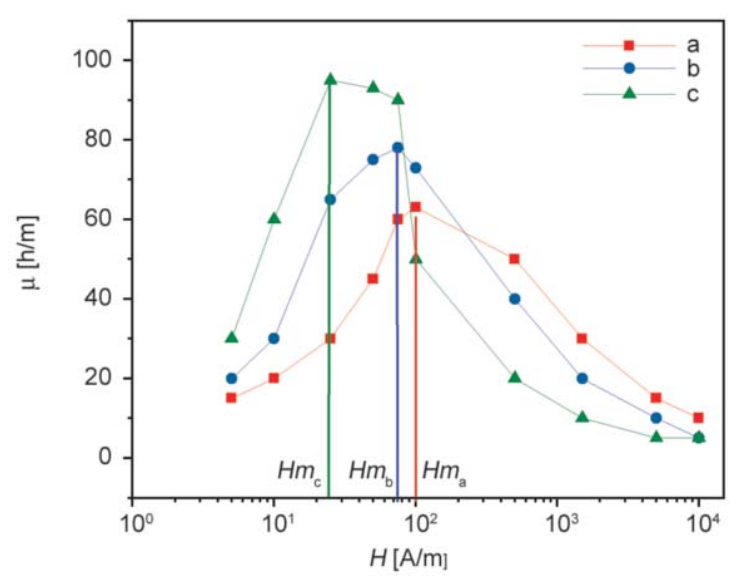

Figure 4. Differential longitudinal magnetic permeability $\mu$ as a function of the applied longitudinal magnetic field $H$ in samples produced under the different experimental conditions (a), (b) and (c), as specified in the caption of Figure 3. The permeability values are generally lower than expected in pure Nickel since the Ni NPs are diluted in the polymeric matrix. Hm indicates the magnetizing field at which the maximum value of $\mu$ is obtained in the three different sample types.
It is evident that when the MFNs are electrospun all around the tube edge without any magnetizing field (case (a)) the differential magnetic permeability is the lowest, and its maximum is reached at the highest magnetizing field value $\left(H m_{\mathrm{a}}\right)$. On the contrary, when the MFNs are deposited in static condition of the manifold - therefore only on the exposed side strip of the tube - and in presence of the field produced by permanent magnets (case (c)), the highest magnetic NPs concentration, and the alignment, correspond to the highest value of $\mu$, moreover the maximum susceptibility is reached at the lowest value of the magnetizing field $\left(H m_{\mathrm{c}}\right)$.

The benefit of the magnetic field application in the alignment of the magnetic particles, and consequently of the fibers which incorporate them, also maintaining manifold rotation (case b), as already shown by the SEM images, is also confirmed by the $\mu$ behavior which is intermediate between case a and c. According to the SEM analysis, the effect of static deposition, which concentrates the magnetic fieldassisted electrospinning on a side strip of the tube and provides larger fibers (Figure 3 curve c), is the most effective in increasing magnetic permeability at low $H$ field.

Considering the morphological and magnetic data, we deduce that the new type of nanocomposite biocompatible tubes, fabricated by a multistage electrospinning technique, corresponds to the pre-established purpose: the dense side strip of MFNs can respond excellently to external magnetic stimuli.

\subsection{MFPTs smart magnetic actuation}

To show that the MFPTs presented here have a remarkable transverse contraction under the action of a moderate magnetic field gradient, the experimental 


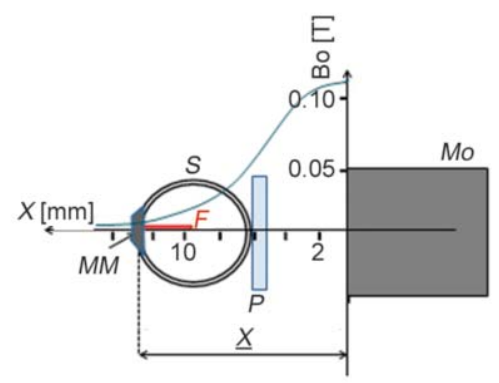

a)

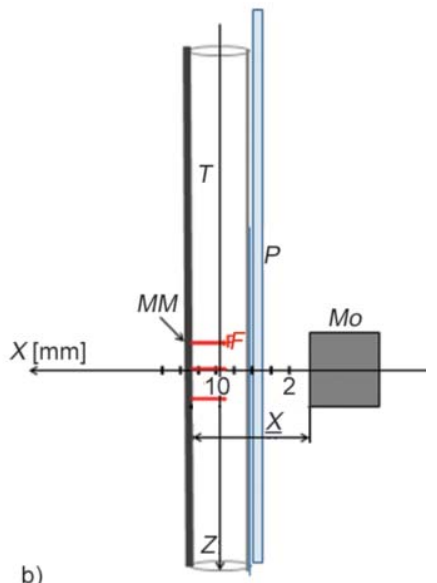

b)

Figure 5. (a) This experimental configuration was designed to observe the deformation of the duct section induced by a transverse magnetic stimulus. The MFPT was placed horizontally on plexiglass support $(P)$, which is parallel to tube axis. The magnetic mat $(M M)$ incorporated in the tube at the terminal section $(S)$ was subjected, along the transverse axis $(X)$, to the magnetic induction field $(B o)$ of a small permanent magnet $(M o)$. The axial Bo intensity behavior (as obtained by a standard hall probe) is shown in the diagram as function of the distance (expressed in millimeter) from the magnet surface. (b) This experimental arrangement was conceived to observe the tube strain by seeing the conduit laterally, as induced by a transverse magnetic stimulus similar to previous case. The tubular sample $(T)$ was kept vertical on the plexiglass support $(P)$ and in the transversal induction field $(B o)$. In this case the magnetic forces $(F)$ are applied to an $M M$ region in the middle of the tube.

arrangement was realized as described in Figure 5. In Figure 5a, to the right, a small cylindrical magnet $M o(\mathrm{NdFeB}$ diameter $=6 \mathrm{~mm}$ and length $=10 \mathrm{~mm})$ and the induction field $B o$ that it produces along its main symmetry axis are reproduced: the blue curve in the figure shows intensity $B o-$ on vertical axis in Tesla unit - with respect to the distance from the magnet $(\underline{X})$. In the same Figure $5 \mathrm{a}$ is schematized the section $(S)$ at the end of an MFPT; the cross-section $(M M)$ of the magnetic fibers ribbon embedded in the tube is visible inside $S$. A plexiglass bar $-P$ supports the tube in a fixed position on the opposite side to MM. A mechanical device produces the micrometric displacement of the magnet so that its distance $\underline{X}$ from the $M M$ ribbon can be reduced, thus increasing the magnetizing field and its $\mathrm{d} B o / \mathrm{d} x$ gradient on the ribbon-shaped magnetic mat incorporated in the polymeric tube.

Figure $5 \mathrm{~b}$ shows in a lateral view the same components of Figure 5a, but in this configuration the magnet $(M o)$ is placed not at the end of the polymer tube, but at an intermediate position, in order to observe the lateral strain of all the tube under the action of the transversal magnetic stimulus. The last can be increased, as in configuration $5 \mathrm{a}$, by decreasing the distance $(\underline{X})$ between the magnet polar surface and the magnetic nanofiber ribbon $(M M)$.
The magnetic mat $(M M)$ inside the conduit is subjected to the $x$ component of the force produced by the permanent magnet (Equation (1)):

$F_{\mathrm{x}}=m_{\mathrm{x}} \frac{\mathrm{d} B O}{\mathrm{~d} x}$

where $m_{\mathrm{x}}$ is the component of the magnetic moment, along the $x$-direction, of the ribbon piece $M M$ immersed in the magnetic field and $\mathrm{d} B o / \mathrm{d} x$ is the $x$ component of the induction field gradient. To increase $F_{\mathrm{x}}$ it is sufficient to bring the magnet closer to the sample. In fact, decreasing the distance $\underline{X}$ between $M M$ and the magnet both $m_{\mathrm{x}}$ and $\mathrm{d} B o / \mathrm{d} x$ increase $-m_{\mathrm{x}}$ increment is due to the increase in the intensity of the induction field $B o-$. This causes an increasing compression of the tube section and the consequent strain as can be verified by the experimental results reported in Figure 6.

Figure 6a reports the images of the final cross-section of the MFPT: in the image on the left (Figure $6 \mathrm{a} 1)$, the tube is outside the magnetizing field (the distance from $M M$ to the magnet is $\underline{X}_{0}=$ $18 \mathrm{~mm}$ ); going to the images on the right, $\underline{X}$ decreases (Figure 6a2) to $\underline{X}_{1}=10 \mathrm{~mm}\left((\mathrm{~d} B / \mathrm{d} x)_{1} \approx 10 \mathrm{~T} / \mathrm{m}\right.$, $\left.B o_{1}=0.015 \mathrm{~T}\right)$ ) and finally (Figure 6a3) to $\underline{X}_{2}=$ $\left.7.0 \mathrm{~mm}(\mathrm{~d} B / \mathrm{d} x)_{2}=30 \mathrm{~T} / \mathrm{m}, B o_{2}=0.03 \mathrm{~T}\right)$. In correspondence, the eccentricity of the cross-section, 

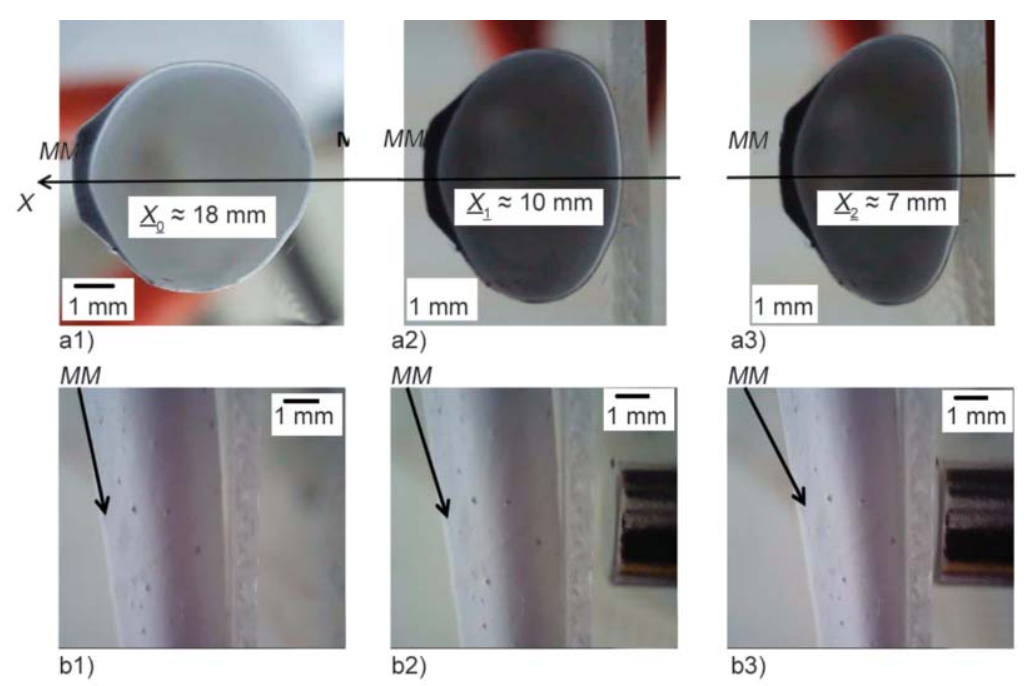

Figure 6. (a) Cross-section contraction of the composite polymer tube due to the application of an increasing both intensity and gradient of the transverse magnetizing field. In case a1 the magnetic ribbon $(M M)$ is at a distance $\underline{X_{0}}=18 \mathrm{~mm}$ at a very low strength and gradient of magnetizing field (see behavior of Bo versus $\underline{X}$ in Figure 5a. In case a2 the distance of the magnet is decreased $\left(\underline{X}_{1}=10 \mathrm{~mm}\right)$; correspondingly the $B o$ field intensity, as well as its gradient, are increased $(B o \approx 15 \mathrm{mT} ; \mathrm{d} B o / \mathrm{d} x \approx 10 \mathrm{mT} / \mathrm{mm})$. In case a3 the magnet distance from $M M$ is the lowest possible (the magnet is practically in contact with the plexiglass holder) the field intensity is about $30 \mathrm{mT}$ and the field gradient approaches the value $30 \mathrm{mT} / \mathrm{mm}$. (b) Transverse contraction of an MFPT, in lateral view, as produced in configuration of Figure $5 \mathrm{~b}$ and in the same sequence of magnetic stimuli applied to the extreme cross-section in case a. In the images b2 and b3 it is visible the magnet and its approaching.

measured as the ratio between the vertical and the horizontal diameters, passes from 1.1 to 1.3 and to 1.7 . The ability to contract under the action of a moderate transverse magnetic field is also evidenced in the experimental configuration in which $B o$ is applied not on the terminal section, but in the central region of the tube (Figure 5b), and its lateral strain is displayed. In Figure $6 \mathrm{~b}$ the sequence b1, b2, and b3 show the lateral view of an MFPT as deformed by the same magnet and at the same distances $\left(\underline{X}_{1}, \underline{X}_{2}\right.$, and $\left.\underline{X}_{3}\right)$ of the sequence a1, a2 and a3, reported in Figure 6a. Also, in this case, the transversal contraction of the tube is evident.

In the cases, $\mathrm{a} 3$ and $\mathrm{b} 3$ in Figure 6, the stress force $F_{\mathrm{x}}$ (Equation (1)) is the maximum possible, since both $m_{\mathrm{x}}$ and $\mathrm{d} B o / \mathrm{d} x$ have the maximum possible value: the MFPT strain produces an increase in the eccentricity of tube section more than $50 \%$.

The effectiveness of the device is based on the presence of the dense magnetic nanofiber ribbon only on a side portion of the tube. This allows having a thin thickness of the not magnetic part of the tube, which confers a very low transverse rigidity, making it possible to produce a large strain by means of a unilateral low pressure. The same effect is not possible by means of a magnetic fiber layer around the entire tube. In fact, the presence of a homogeneous magnetic shell confers a higher thickness to the duct section combined with a higher rigidity of the composite material; so that when the same moderate magnetic field is applied as in the arrangements of Figure 5, the addition of both magnetic forces around the section contour and attractive forces among magnetized parts of the section (in particular between the opposite sides along $X$-axis) does not compensate for the increase in transverse rigidity.

The easy and noteworthy transversal contraction demonstrated here experimentally - seems very promising to be applied in several practical cases, being an alternative or an evolution with respect existent methodology or devices, as will be outlined in the conclusions.

\section{Conclusions}

The combination of electrospinning on a rotating manifold and in static condition - assisted by a magnetic field- has been designed and realized so as to produce PCU nanofiber tubes containing along a side a ribbon of longitudinally aligned nanofiber incorporating Ni nanoparticles. The morphological and magnetic differences with similar polymeric tubes incorporating magnetic nanofibers homogeneously aligned, and without preferential localization, are experimentally evidenced and show the improvement obtained by the use of the new methodology. The new design composite ducts are particularly effective 
for a smart response (over 50\% increase in eccentricity of the tube cross-section) when, at low magnetic field intensity $(\approx 30 \mathrm{mT})$, a medium intensity gradient of transversal magnetic field $(30 \mathrm{mT} / \mathrm{mm})$ is applied: the high transverse contraction is demonstrated by the vision of both cross-section and the lateral view of the tube. The transverse strain is reversible and can be enhanced by increasing the magnetic stimulus intensity. The fabrication process is easy to reproduce and reliable. Moreover, also if currently the used methodology appears not suitable for massproduction, to achieve these purpose process improvements are predictable by implementation with other methodologies, for example, the bubble electrospinning or the continuous filament electrospinning [33, 49].

The smart properties demonstrated by the new MFPTs are notable for potential application in biomedical devices when a controlled transverse contraction can resolve occlusive phenomena in conduit substitutes of small or medium caliber [50], as for example avoiding periscope approach in vascular prosthesis [51] or to solve obstruction problems in cardiology [52-56] and even to help insert guidewires and probes [57-59]. Severe obstruction problems are also recurrent in the urinary [60], biliary [61], bowel [62-64], lacrimal [65] and esophagogastic [66] ducts. In these cases, in addition to standard surgical procedures, the stent is often used [67-69] or laser treatment has been tried [70]. Only recently, the compression induced by magnets [71] or the magnetically induced vibration of a metal element [72] has been successfully applied. The magnetically functionalized polymer tubes, presented in this study, represent an important evolution of these latter devices, with the advantage of not needing metal parts or magnets - incorporated into the patient's body - to favor drainage or expulsion of obstructions - since only a magnetic stimulus coming from the outside is sufficient. The innovative MFPTs can be previously inserted where their function is needed, like an intelligent stent able to provide a great transversal contraction. On the other side, due the same properties of not requiring components inside the tube, the developed MFPTs appear useful even outside the biomedical ambient, wherever it is important to control a flow of a fluid in conduit circulation, in particular for micro-electromechanical devices such as fluid flow gates [73]. Finally, many other applications can be identified in perspective for such materials that are elastic and deformable on command and also conductive, as for example flexible sensors, EMI shielding and piezoelectric devices [74-76].

\section{Acknowledgements}

'Vincenzo Guarino, Vincenzo Iannotti, Giovanni Ausanio contributed equally to this work.' The authors are grateful to Dr. Luca Lanotte (INRA, Science et Technologie du Lait et de l'Oeuf 65, rue de Saint Brieuc - 35042 Rennes Cedex, France) for stimulating discussion of the results and useful suggestions.

\section{References}

[1] Li J., He J-H.: Fabrication and characterization of $\mathrm{ZrO}_{2}$ nanofibers by critical bubble electrospinning for hightemperature-resistant adsorption and separation. Absorption Science and Technology, 37, 425-437 (2019). https://doi.org/10.1177/0263617419828268

[2] Yu L., Shao Z., Xu L., Wang M.: High throughput preparation of aligned nanofibers using an improved bubbleelectrospinning. Polymers, 9, 658/1-658/9 (2017). https://doi.org/10.3390/polym9120658

[3] Tian D., He J-H.: Macromolecular electrospinning: Basic concept \& preliminary experiment. Results in Physics, 11, 740-742 (2018). https://doi.org/10.1016/j.rinp.2018.10.042

[4] Blond D., Walshe W., Young K., Blighe F. M., Khan U., Almecija D., Carpenter L., McCauley J., Blau W. J., Coleman J. N.: Strong, tough, electrospun polymernanotube composite membranes with extremely low density. Advanced Functional Materials, 18, 2618-2624 (2008). https://doi.org/10.1002/adfm.200701487

[5] Huang C., Soenen S. J., Rejman J., Trekker J., Chengxun L., Lagae L., Ceelen W., Wilhelm C., Demeester J., De Smedt S. C.: Magnetic electrospun fibers for cancer therapy. Advanced Functional Materials, 12, 2479-2486 (2012). https://doi.org/10.1002/adfm.201102171

[6] Chen C., Tang J., Gu Y., Liu L., Liu X., Deng L., Martins C., Sarmento B., Cui W., Chen L.: Bioinspired hydrogel electrospun fibers for spinal cord regeneration. Advanced Functional Materials, 29, 1806899/1-1806899/11 (2019). https://doi.org/10.1002/adfm.201806899

[7] Zhang L., Yu W., Han C., Guo J., Zhang Q., Xie H., Shao Q., Sun Z., Guod Z.: Large scaled synthesis of heterostructured electrospun $\mathrm{TiO}_{2} / \mathrm{SnO}_{2}$ nanofibers with an enhanced photocatalytic activity. Journal of the Electrochemical Society, 164, H651-H656 (2017). https://doi.org/10.1149/2.1531709jes 
[8] Guo Y., Yang X., Ruan K., Kong J., Dong M., Zhang J., Gu J., Guo Z.: Reduced graphene oxide heterostructured silver nanoparticles significantly enhanced thermal conductivities in hot-pressed electrospun polyimide nanocomposites. ACS Applied Material Interfaces, 11, 25465-25473 (2019).

https://doi.org/10.1021/acsami.9b10161

[9] Haider A., Haider S., Kang I-K.: A comprehensive review summarizing the effect of electrospinning parameters and potential applications of nanofibers in biomedical and biotechnology. Arabian Journal Chemistry, 11, 1165-1188 (2018).

https://doi.org/10.1016/j.arabjc.2015.11.015

[10] Fiqranti I. A., Widiyanti P., Manaf M. A., Savira C. Y., Cahyani N. R., Bella F. R.: Poly-L-lactic acid (PLLA)chitosan-collagen electrospun tube for vascular graft application. Journal of Functional Biomaterials, 9, 32 44 (2018).

https://doi.org/10.3390/jfb9020032

[11] Khan M. Q., Kharaghani D., Nishat N., Sanaullah, Shahzad A., Yamamoto T., Inoue Y., Kim Y. S.: In vitro assessment of dual-network electrospun tubes from poly (1,4 cyclohexane dimethylene isosorbide terephthalate)/PVA hydrogel for blood vessel application. Journal of Applied Polymer Science, 136, 47222/1-47222/6 (2019). https://doi.org/10.1002/app.47222

[12] Moazeni N., Semmani D., Rafeinia M., Hasani H., Naeimi M., Sadrjahani M.: The effect of electrospinning parameters on the compliance behavior of electrospun polyurethane tube for artificial common bile duct. Polymer Science Series A, 59, 67-75 (2017).

https://doi.org/10.1134/S0965545X17010114

[13] Son S-R., Franco R-A., Bae S-H., Min Y-K., Lee B-T.: Electrospun PLGA/gelatin fibrous tubes for the application of biodegradable intestinal stent in rat model. Journal of Biomedical Materials Research Part B: Applied Biomaterials, 101, 1095-1105 (2013).

https://doi.org/10.1002/jbm.b.32923

[14] Li C., Wu P-M., Han J., Ahn C. H.: A flexible polymer tube lab-chip integrated with microsensors for smart microcatheter. Biomedical Microdevices, 10, 671-679 (2008). https://doi.org/10.1007/s10544-008-9178-3

[15] Chaloupka K., Motwani M., Seifalian A. M.: Development of a new lacrimal drainage conduit using POSS nanocomposite. Biotechnology and Applied Biochemistry, 58, 363-386 (2011).

https://doi.org/10.1002/bab.53

[16] Cirillo V., Clements B. A., Guarino V., Bushman J., Kohn J., Ambrosio L.: A comparison of the performance of mono- and bi-component electrospun conduits in a rat sciatic model. Biomaterials, 35, 8970-8982 (2014).

https://doi.org/10.1016/j.biomaterials.2014.07.010
[17] Evrova O., Houska J., Welti M., Bonavoglia E., Calcagni M., Giovanoli P., Vogel V., Bushmann J.: Bioactive, elastic, and biodegradable emulsion electrospun degrapol tube delivering PDGF-BB for tendon rupture repair. Macromolecular Bioscience, 16, 1048-1075 (2016).

https://doi.org/10.1002/mabi.201500455

[18] Bürgisser G. M., Calcagni M., Müller A., Bonavoglia E., Fessel G., Snedeker J. S., Giovanoli P., Bushmann J.: Prevention of peritendinous adhesions using an electrospun degrapol polymer tube: A histological, ultrasonographic, and biomechanical study in rabbits. BioMed Research International, 2014, 656240/1-656240/11 (2014).

https://doi.org/10.1155/2014/656240

[19] Liu W., Nie M., Wang Q.: Biaxial reinforcements for polybutene-1 medical-tubes achieved via flow-design controlled morphological development of incorporated polystyrene: In-situ microfibrillation, alignment manipulation and performance optimization. Composites Science and Technology, 119, 124-130 (2015).

https://doi.org/10.1016/j.compscitech.2015.10.007

[20] Singha P., Locklin J., Handa H.: A review of the recent advances in antimicrobial coatings for urinary catheters. Acta Biomaterialia, 50, 20-40 (2017).

https://doi.org/10.1016/J.actbio.2016.11.070

[21] Ruiz J. A. R., Sanjuán A. M., Vallejos S., Garcia F. C., Garcia J. M.: Smart polymers in micro and nano sensory devices. Chemosensors, 6, 12/1-12/20 (2018). https://doi.org/10.3390/chemosensors6020012

[22] Kim Y-J., Matsunaga Y. T.: Thermo-responsive polymers and their application as smart biomaterials. Journal of Materials Chemistry B, 5, 4307-4321 (2017). https://doi.org/10.1039/C7TB00157F

[23] Chen H., Xia H., Qiu Y., Xu Z., Ni Q-Q.: Smart composites of piezoelectric particles and shape memory polymers for actuation and nanopositioning. Composites Science and Technology, 163, 123-132 (2018). https://doi.org/10.1016/j.compscitech.2018.05.004

[24] Chakraborty D. D., Nath L. K., Chakraborty P.: Recent progress in smart polymers: Behavior, mechanistic understanding and application. Polymer-Plastics Technology and Engineering, 57, 945-957 (2018). https://doi.org/10.1080/03602559.2017.1364383

[25] Yin L., Wang S., Zuo S.: Water-jet outer sheath with braided shape memory polymer tubes for upper gastrointestinal tract screening. The International Journal of Medical Robotics and Computer Assisted Surgery, 14, e1944/1-e1944/11 (2018). https://doi.org/10.1002/rcs.1944

[26] Wang Y., Liu J., Zhu D., Chen H.: Active tube-shaped actuator with embedded square rod-shaped ionic polymer-metal composites for robotic-assisted manipulation. Applied Bionics and Biomechanics, 2018, 4031705/1-4031705/12 (2018). https://oi.org/10.1155/2018/4031705 
[27] Tsugawa M. A., Leang K. K., Palmre V., Kim K. J.: Sectored tube-shaped ionic polymer-metal composite actuator with integrated sensor. in 'ASME 2013 Conference on Smart Materials, Adaptive Structures and Intelligent Systems, Snowbird, USA' Vol 2, V002T06A001/1-V002T06A001/7 (2014). https://doi.org/10.1115/SMASIS2013-3017

[28] Du H., Liu L., Zhang F., Zhao W., Leng J., Liu Y.: Thermal-mechanical behavior of styrene-based shape memory polymer tubes. Polymer Testing, 57, 119-125 (2017). https://doi.org/10.1016/j.polymertesting.2016.11.011

[29] Raghavan S. R., Fernandes N. J., Cipriano B. H.: Shapechanging tubular hydrogels. Gels, 4, 18/1-18/10 (2018). https://doi.org/10.3390/gels4010018

[30] Guarino V., Ausanio G., Iannotti V., Ambrosio L., Lanotte L.: Electrospun nanofiber tubes with elastomagnetic properties for biomedical use. Express Polymer Letters 12, 318-329 (2018).

https://doi.org/10.3144/expresspolymlett.2018.28

[31] Wu Y., Yu J-Y., He Y-H., Wan Y-Q.: Controlling stability of the electrospun fiber by magnetic field. Chaos, Solitons and Fractals, 32, 5-7 (2007).

https://doi.org/10.1016/j.chaos.2006.05.023

[32] Guarino V., Iannotti V., Ausanio G., Ambrosio L., Lanotte L.: Elastomagnetic nanofiber wires by magnetic field assisted electrospinning. Express Polymer Letters 13, 419-428 (2019).

https://doi.org/10.3144/expresspolymlett.2019.35

[33] Lach A. A., Morris H. L., Martins J. A., Stace E. T., Carr A. J., Mouthuy P-A.: Pyridine as an additive to improve the deposition of continuous electrospun filaments. PLOS ONE, 14, e0214419/1-e0214419/16 (2018). https://doi.org/10.1371/journal.pone.0214419

[34] Li Y., Zhang C., Zhu L-F., Ahmad Z., Li J-S., Chang M-W.: Elastic antibacterial membranes comprising particulate laden fibers for wound healing applications. Journal of Applied Polymer Science, 136, 47105/147105/9 (2019). https://doi.org/10.1002/app.47105

[35] Li Y., Zhang T., Jiang B., Zhao L., Liu H., Zhang J., Fan J., Guo Z., Huang Y.: Interfacially reinforced carbon fiber silicone resin via constructing functional nanostructural silver. Composites Science and Technology, 181, 107689/1-107689/6 (2019).

https://doi.org/10.1016/j.compscitech.2019.107689

[36] Zhang Z., Zhang J., Li S., Liu J., Dong M., Li Y., Lu N., Lei S., Tang J., Fan T., Guo Z.: Effect of graphene liquid crystal on dielectric properties of polydimethylsiloxane nanocomposites. Composites Part B: Engineering, 176, 107338/1-107338/9 (2019).

https://doi.org/10.1016/j.compositesb.2019.107338

[37] Stevenson Jr. A. T., Reese L. M., Hill T. K., McGuire J., Mohs A. M., Shekhar R., Bichford L. R., Whittington A. R.: Fabrication and characterization of medical grade polyurethane composite catheters for near-infrared imaging. Biomaterials, 54, 168-176 (2015).

https://doi.org/10.1016/j.biomaterials.2015.03.020
[38] Gostev A. A., Karpenko A. A., Laktionov P. P.: Polyurethanes in cardiovascular prosthetics. Polymer Bulletin, 75, 4311-4325 (2018).

https://doi.org/10.1007/s00289-017-2266-x

[39] Arjun G. N., Ramesh P.: Structural characterization, mechanical properties, and in vitro cytocompatibility evaluation of fibrous polycarbonate urethane membranes for biomedical applications. Journal of Biomedical Materials Research A, 100, 3042-3050 (2012). https://doi.org/10.1002/jbm.a.34255

[40] Sobczak M.: Biodegradable polyurethane elastomers for biomedical applications - Synthesis methods and properties. Polymer-Plastics Technology and Engineering, 54, 155-172 (2015).

https://doi.org/10.1080/03602559.2014.955201

[41] Zhang D., Karki A. B., Rutman D., Young D. P., Wang A., Cocke D., Ho T. H., Guo Z.: Electrospun polyacrylonitrile nanocomposite fibers reinforced with $\mathrm{Fe}_{3} \mathrm{O}_{4}$ nanoparticles: Fabrication and property analysis. Polymer, 50, 4189-4198 (2009).

https://doi.org/10.1016/j.polymer.2009.06.062

[42] Wang L., Qiu H., Liang C., Song P., Han Y., Han Y., Gu J., Kong J., Pan D., Guo Z.: Electromagnetic interference shielding MWCNT-Fe $\mathrm{O}_{4} @ \mathrm{Ag} /$ epoxy nanocomposites with satisfactory thermal conductivity and high thermal stability. Carbon, 141, 506-514 (2019). https://doi.org/10.1016/j.carbon.2018.10.003

[43] Li Y., Jing T., Xu G., Tian J., Dong M., Shao Q., Wang B., Wang Z., Zheng Y., Yang C., Guo Z.: 3-D magnetic graphene oxide-magnetite poly(vinyl alcohol) nanocomposite substrates for immobilizing enzyme. Polymer, 149, 13-22 (2018).

https://doi.org/10.1016/j.polymer.2018.06.046

[44] Ausanio G., Hison C. L., Iannotti V., Lanotte Jr. L., Lanotte L.: Magneto-piezoresistance in elastomagnetic composites. Journal of Applied Physics, 110, 063903/1063903/9 (2011).

https://doi.org/10.1063/1.3634120

[45] Lanotte L., Ausanio G., Iannotti V., Pepe G., Carotenuto G., Netti P., Nicolais L.: Magnetic and magnetoelastic effects in a composite material of Ni microparticles in a silicone matrix. Physical Review B, 63, 054438/1054438/6 (2001).

https://doi.org/10.1103/PhysRevB.63.054438

[46] Ausanio G., Iannotti V., Lanotte Jr. L., Lanotte L.: Optimization of the coupling between piezoresistivity and magnetoelasticity in an elastomagnetic composite to sense a spatial gradient of the magnetic field. The $\mathrm{Eu}-$ ropean Physical Journal B, 86, 51-55 (2013). https://doi.org/10.1140/epjb/e2012-30657-1

[47] Guarino V., Branda F., Ausanio G., Iannotti V., Lanotte L., Ambrosio L.: Elastomagnetic NI-PDMS nanofibers via coaxial electrospinning. Materials Research Express, 5, 085029/1-085029/7 (2018). https://doi.org/10.1088/2053-1591/aad31c 
[48] Iannotti V., Amoruso S., Ausanio G., Wang X., Lanotte L., Barone A. C., Margaris G., Trohidou K. N., Fiorani D.: Interplay between particle anisotropy and exchange interaction in Fe nanoparticle films. Physical Review B, 83, 214422/1-214422/5 (2011). https://doi.org/10.1103/PhysRevB.83.214422

[49] Chen R-X., Li Y., He J-H.: Mini-review on bubbfil spinning process for mass-production of nanofibers. Matéria (Rio De Janeiro), 19, 325-344 (2014). https://doi.org/10.1590/S1517-70762014000400002

[50] Ahn H., Ju Y. M., Takahashi H., Williams D. F., Yoo J. J., Lee S. J., Okano T., Atala A.: Engineered small diameter vascular grafts by combining cell sheet engineering and electrospinning technology. Acta Biomateralia, 16, 14-22 (2015).

https://doi.org/10.1016/j.actbio.2015.01.030

[51] Drexel T., Helmer G., Garcia S., Raveendran G.: Management of left main coronary artery obstruction after transcatheter aortic valve replacement utilizing a periscope approach. Catheterization Cardiovascular Interventions, Valvular and Structural Heart Diseases, 92, 1444-1448 (2018). https://doi.org/10.1002/ccd.27317

[52] Nappi F., Spadaccio C., Sablayrolles J-L.: Delayed prosthesis malposition after transcatheter aortic valve implantation causing coronaries obstruction. European Journal of Cardio-Thoracic Surgery, 52, 1227-1228 (2017).

https://doi.org/10.1093/ejcts/ezx266

[53] Kumar A., Sharma P., Arora A.: Review article: Portal vein obstruction - Epidemiology, pathogenesis, natural history, prognosis and treatment. Aliment Pharmacology and Therapeutics, 41, 276-292 (2015).

https://doi.org/10.1111/apt.13019

[54] Scandroglio A. M., Kausfmann F., Pierri M., Kretzschmar A., Müller M., Pergantis P., Dreysse S., Falk V., Krabatsch T., Potapov E. V.: Diagnosis and treatment algorithm for blood flow obstructions in patients with left ventricular assist device. Journal of the American College of Cardiology, 67, 2758-2768 (2016). https://doi.org/10.1016/j.jacc.2016.03.573

[55] Wang S., Culi H., Yu Q., Chen H., Zhu C., Wang J., Xiao M., Zhang Y., Wu R., Hu S.: Excision of anomalous muscle bundles as an important addition to extended septal myectomy for treatment of left ventricular outflow tract obstruction. The Journal of Thoracic and Cardiovascular Surgery, 152, 461-468 (2016).

https://doi.org/10.1016/j.jtcvs.2016.01.051

[56] van Vuren T. M. A. J., van Laanen J. H. H., de Geus M., Nelemans P. J., de Graaf R., Wittens C. H. A.: A randomised controlled trial comparing venous stenting with conservative treatment in patients with deep venous obstruction: Research protocol. BMJ Open, 7, e017233/1-2017233/8 (2017).

https://doi.org/10.1136/bmjopen-2017-017233
[57] Berry R. B., Han J. Y., Tabibian J. H.: Difficult biliary cannulation: Historical perspective, practical updates, and guide for the endoscopist. World Journal of Gastrointestinal Endoscopy, 11, 5-21 (2019).

https://doi.org/10.4253/wjge.v11.i1.5

[58] Khashab M. A., Bukhari M., Baron T. H., Nieto J., El Zein M., Chen Y-I., Chavez Y. H., Ngamruengphong S., Alawad A. S., Kumbhari V., Itoi T.: International multicenter comparative trial of endoscopic ultrasonographyguided gastroenterostomy versus surgical gastrojejunostomy for the treatment of malignant gastric outlet obstruction. Endoscopy International Open, 5, E275E281 (2017).

https://doi.org/10.1055/s-0043-101695

[59] Rowland K. J., Cunningham A. J., Jazrawi S. F., Prauner R., Butler M. W., Jafri M.: Novel application of SpyGlass $^{\mathrm{TM}}$ cholangioscopy in the diagnosis and treatment of extrahepatic biliary obstruction in infants. Journal of Pediatric Surgery Case Reports, 38, 19-22 (2018). https://doi.org/10.1016/j.epsc.2018.07.028

[60] Xu N., Chen S-H., Xue X-Y., Zheng Q-S., Wei Y., Jiang T., Li X-D., Huang J-B., Cali H.: Comparison of retrograde balloon dilatation and laparoscopic pyeloplasty for treatment of ureteropelvic junction obstruction: Results of a 2-year follow-up. PLOS ONE, 11, e0152463/1e0152463/8 (2016). https://doi.org/10.1371/journal.pone.0152463

[61] Inamdar S., Slattery E., Bhalla R., Sejpal D. V., Trindade A. J.: Comparison of adverse events for endoscopic vs percutaneous biliary drainage in the treatment of malignant biliary tract obstruction in an inpatient national cohort. JAMA Oncology, 2, 112-117 (2016). https://doi.org/10.1001/jamaoncol.2015.3670

[62] Patel R., Borad N. P., Merchant A. M.: Comparison of outcomes following laparoscopic and open treatment of emergent small bowel obstruction: An 11-year analysis of ACS NSQIP. Surgical Endoscopy, 32, 4900-4911 (2018). https://doi.org/10.1007/s00464-018-6249-2

[63] Lorentzen L., Øines M. N., Oma E., Jensen K. K., Kristian K., Jorgensen L. N.: Recurrence after operative treatment of adhesive small-bowel obstruction. Journal of Gastrointestinal Surgery, 22, 329-334 (2018). https://doi.org/10.1007/s11605-017-3604-x

[64] Seyer-Hansen M., Engekvist A., Forman A., Riiskjaer M.: Risk of bowel obstruction during in vitro fertilization treatment of patients with deep infiltrating endometriosis. Acta Obstetricia and Gynecologica Scandinava, 97, 47-52 (2018). https://doi.org/10.1111/aogs.13253

[65] Gupta N., Neeraj C., Smriti B., Sima D.: A comparison of the success rates of endoscopic-assisted probing in the treatment of membranous congenital nasolacrimal duct obstruction between younger and older children and its correlation with the thickness of the membrane at the Valve of Hasner. The International Journal on Orbital Disorders, Oculoplastic and Lacrimal Surgery, 37, 257-261 (2018).

https://doi.org/10.1080/01676830.2017.1383483 
[66] Clayton S. B., Patel R., Richter J. E.: Functional and anatomic esophagogastic junction outflow obstruction: Manometry, timed barium esophagram findings, and treatment outcomes. Clinical Gastroenterology and Hepatology, 14, 907-911 (2016).

https://doi.org/10.1016/j.cgh.2015.12.041

[67] Tan G. M., Chi K. W. K., Yan B. P. Y.: Mid-term results of a novel dedicated venous stent for the treatment of chronic thoracic central vein obstruction of benign aetiology. European Journal of Vascular and Endovascular Surgery, 57, 417-423 (2019).

https://doi.org/10.1016/j.ejvs.2018.10.009

[68] Ribeiro I. B., Bernardo W. M., da Costa Martins B. D., de Moura D. T. H., Baba E. R., Josino I. R. R., Miyajima N. T., Cordero M. A. C., de Carvalho Visconti T. A. D., Ide E., Sakai P., de Moura E. G. H.: Colonic stent versus emergency surgery as treatment of malignant colonic obstruction in the palliative setting: A systematic review and meta-analysis. Endoscopy International Open, 6, E558-E566 (2018).

https://doi.org/10.1055/a-0591-2883

[69] Garcia-Cano J., Yasuda-Gastello M. K., Taberna-Arana L., Godoy-Loópez M. A.: Mo1118 metal stents as palliative treatment in frail elderly patients with biliary obstruction due to difficult common bile duct stones. Gastrointestinal Endoscopy, 87, AB405 (2018).

https://doi.org/10.1016/j.gie.2018.04.1879

[70] Hsu F-S., Chou C-W., Chang H-C., Tu Y-P., Sha S-J., Chung H-H., Huang K-H.: Comparison of multipulse laser vaporesection versus plasmakinetic resection for treatment of benign prostate obstruction. Scientific Reports, 9, 6427/1-6427/8 (2019). https://doi.org/10.1038/s41598-019-42903-6
[71] Parlak E., Koksal A. S., Kucukay F., Eminler A. T., Toka B., Uslan M. I.: A novel technique for the endoscopic treatment of complete biliary anastomosis obstructions after liver transplantation: Through-the-scope magnetic compression anastomosis. Gastrointestinal Endoscopy, 85, 841-847 (2017).

https://doi.org/10.1016/j.gie.2016.07.068

[72] Park H., Raffiee A. H., John S. W. M., Anderkani A. M., Lee H.: Towards smart self-clearing glaucoma drainage device. Microsysthem and Nanoengeenering, 35, 35/1-35/12 (2018). https://doi.org/10.1038/s41378-018-0032-3

[73] Glaser R., Caccese V., Shahinpoor M.: Development of novel smart MR-gates for wireless dynamic control of fluid flow. Smart Materials and Structures, 22, 015012/1-015012/17 (2013).

https://doi.org/10.1088/0964-1726/22/1/015012

[74] Chen J., Yu Q., Cui X., Dong M., Zhang J., Wang C., Fan J., Zhu Y., Guo Z.: An overview of stretchable strain sensors from conductive polymer nanocomposites. Journal of Materials Chemistry C, 7, 11710-11730 (2019). https://doi.org/10.1039/C9TC03655E

[75] Jiang D., Wang Y., Li B., Sun C., Wu Z., Yan H., Xing L., Qi S., Li Y., Liu H., Xie W., Wang X., Ding T., Guo Z.: Flexible sandwich structural strain sensor based on silver nanowires decorated with self-healing substrate. Macromolecular Materials and Engineering, 304, 1900074/1-1900074/9 (2019). https://doi.org/10.1002/mame.201900074

[76] Wei H., Wang H., Xia Y., Cui D., Shi Y., Dong M., Liu C., Ding T., Zhang J., Ma Y., Wang N., Wang Z., Sun Y., Wei R., Guo Z.: An overview of lead-free piezoelectric materials and devices. Journal of Materials Chemistry C, 6, 12446-12467 (2018). https://doi.org/10.1039/C8TC04515A 